and the consequent risk of a heightened prevalence of febrile seizure recurrence emphasizes the importance of parental education in the management of fever and febrile seizures. In the absence of a safe and satisfactory alternative to phenobarbital, parental anxiety may be allayed by the prescription of intermittent prophylactic treatment with diazepam at times of fevers but poor compliance minimizes its effectiveness in practice. The home use of rectal diazepam, employed more frequently in Europe than in the U.S., may offer an alternative method of parental involvement in selected cases.

HYPEREXPLEXIA OR HEREDITARY STIFF BABY SYNDROME

The stiff baby syndrame and its diagnostic distinction from epilepsy is reviewed from the Service de Neuronatologie, Pavillon de la Mere et l'Enfant, Nantes, France. The disease hyperexplexia was first described in 1962 by Kok and Bruyn in 29 members of one family and occurred as a dominant autosomal transmission. It was distinguished by a permanent hypertonia that is heightened by the slightest stimulus. The hypertonia was noted at birth and became less pronounced during the first year of life but later could lead to repeated falls. The electromyogram showed persistent activity even at rest and the activity was abolished by diazepam. Lingam $S$, Wilson $J$, and Hart $E$ named the condition "hereditary stiff baby syndrome" (Am J Dis Child 1981; 135:909). The child has a fixed stare and an expression of anxiety. The hypertonia diminishes during sleep and increases with the slightest psychic or tactile stimulus. Attacks of hypertonia may involve respiratory muscles and lead to apneas which can endanger the child's life. Digestive disorders including vomiting are usually associated with a hiatal hernia. The electroencephalogram is normal. (Tohier $\mathrm{C}$ et al. Hyperexplexia or stiff baby syndrame. Arch Dis Child April 1991; 66: 460-461).

COMMENT. In addition to myoclonic epilepsy, the differential diagnosis includes the stiff man syndrome which is not hereditary but which may occur in children (Millichap JG, unpublished observation), the Isaacs-Mertens syndrome with distal hypertonia and fasciculations, the jumping Frenchmen of Maine syndrome with violent starts induced by slight stimuli and associated with echolalia and echopraxia, and Gilles de la Tourette syndrome. The pathology of stiff man syndrome has been localized to the spinal interneurons but the mechanism of hyperexplexia is controversial. Treatment with diazepam is effective.

POLYPHAFMAOOTHERAPY IN INSTITUTIONALIZED EPILEPTIC OHILDREN An at tempt to minimize polypharmacotherapy, to discont inue the use of phenobarbital, and to assess the relation between drug levels and antiepileptic effect in institutionalized severely retarded children is reported from the Department of Pediatrics, St. Goran's Hospital, Stockholm, and the Division of Clinical Pharmacology, University Hospital, Uppsala, Sweden. Nine severely mentally retarded patients 
with severe epilepsy were studied during a three year period by a multidisciplinary team. Combination therapy with two or three drugs gave the best antiepileptic effect and monotherapy was possible in only two patients. Phenobarbital withdrawal was possible in two of four patients. Regular phenytoin serum monitoring was necessary to avoid high levels that could be unnoticed for long periods and possibly result in chronic long-term side effects. (Ferngren $\mathrm{H}$ et al. Mono- or polypharmacotherapy in institutionalized epileptic children with severe mental retardation? A team approach for optimizing antiepileptic therapy. Acta Paediatr Scand April 1991; 80:458-465).

COMMENT. The tendency among neurologists to attempt conversion from polytherapy to monotherapy in severely retarded institutionalized patients may be hazardous and inadvisable. This study has demonstrated that polytherapy is frequently required. The recognition of anticonvulsant side effects may be more difficult in retarded patients compared to clinic patients and frequent serum drug monitoring is important.

\section{COMBINATION PHENOBARBITAL/ANTIBIOTIC DRUG REACTIONS}

Undesirable drug reactions during simultaneous administration of high dosage phenobarbital and beta lactam antibiotics, mainly Cefotaxim, are reported in 24 of 49 children admitted to intensive care at the Klinikum der J W Goethe Universitat, Frankfort, Germany. The reactions were mainly exanthematous skin rashes which in some cases progressed to Stevens-Johnson syndrame. (Harder S et al. Unerwunschte Arzneimittelreaktionen bei gleichzeitiger Gabe von hochdosiertem Phenobarbital und Betalaktam-Antibiotika. Kl in Padiatr Nov/Dez 1990; 202: 404-407).

COMMENT. The incidence of skin reaction with phenobarbital is relatively rare compared to the anticonvulsants phenytoin and carbamazepine but the risk of serious skin reactions may be increased by the simultaneous administration of the antibiotic Cefotaxim.

\section{TREATMENT OISET AND EPILEPSY PROGNOSIS}

The efficacy of treatment in relation to the lost time "tiempo perdido" in a group of 3529 epileptic patients was evaluated in the Division of Neurology and Clinical Neurophysiology, Hospital General du Cataluna, Barcelona, Spain. The mean follow-up period was ten years. The "lost time" is the period elapsed fram the onset of symptams and the beginning of long-term anticonvulsant treatment. In 970 patients with a lost time of less than a year, $86 \%$ were seizure-free whereas in 922 patients whose treatment was delayed greater than 11 years, $64 \%$ were seizure-free. Delay in starting anticonvulsant medication influenced the success of drug withdrawal. Of 710 patients who discontinued treatment, 315 (44\%) had treatment initiated within one year of onset of seizures whereas drug withdrawal was possible in only 106 (15\%) of the group in which treatment was delayed over 11 years. A total of 144 $(20 \%)$ patients had seizure recurrences after drug withdrawal and of 